\title{
Mapping prominence plasma parameters from eclipse observations
}

\author{
Sonja Jejčič ${ }^{1}$, Petr Heinzel ${ }^{2}$, Maciej Zapiór ${ }^{2,3}$, Miloslav Druckmüller ${ }^{4}$, \\ Stanislav Gunár ${ }^{2}$, and Pavel Kotré ${ }^{2}$ \\ ${ }^{1}$ University of Ljubljana, Department of Physics, Ljubljana, Slovenia \\ email: sonja.jejcic@guest.arnes.si \\ ${ }^{2}$ Astronomical institute of the Academy of Sciences, Ondřejov, Czech Republic \\ email: pheinzel@asu.cas.cz, email: gunar@asu.cas.cz email: pkotrc@asu.cas.cz \\ ${ }^{3}$ Departament de Física, Universitat de les Illes Balears, Palma de Mallorca, Spain \\ email: maciej.zapior@uib.es \\ ${ }^{4}$ Institute of Mathematics, Brno University of Technology, Brno, Czech Republic \\ email: druckmuller@fme.vutbr.cz
}

\begin{abstract}
Using the eclipse observations, we construct the maps of quiescent prominence temperatures, electron densities, pressures and geometrical thicknesses. For this we use the RGB signal of prominence visible-light emission detected during the total solar eclipse on August 1, 2008 in Mongolia, and quasi-simultaneous $\mathrm{H} \alpha$ spectra taken at Ondřejov observatory. The method of disentangling the electron density and effective geometrical thickness was described by Jejčič \& Heinzel (2009) and is used here for the first time to analyse the spatial variations of various prominence parameters.
\end{abstract}

Keywords. Eclipse Observations; Prominences, Quiescent; Spectral Line, Intensity and Diagnostics

\section{Determination of temperature and electron densities from eclipse observations}

In this study we used the $\mathrm{H} \alpha$ line spectral observations of a quiescent prominence together with RGB eclipse observations from August 1, 2008 (Figure 1). After coalignment between $\mathrm{H} \alpha$ line spectra and visible-light prominence from the eclipse observations, we used calibrated $\mathrm{H} \alpha$ profiles to compute the temperature. The temperature is derived from simple relation $\Delta \lambda_{\mathrm{D}}=F \cdot E_{\mathrm{H} \alpha} /\left(I_{\max } \sqrt{\pi}\right)$, where $\Delta \lambda_{\mathrm{D}}$ is the Doppler width (in $\AA$ ), $E_{\mathrm{H} \alpha}$ the $\mathrm{H} \alpha$ integrated intensity and $I_{\max }$ the maximal (peak) intensity of the $\mathrm{H} \alpha$ emission profile. $F$ is the opacity correction factor (Heinzel et al. (1994)). The kinetic temperature is derived assuming a uniform value of the microturbulent velocity $5 \mathrm{~km} \mathrm{~s}^{-1}$. The electron density was determined using a generalized approach of Jejčič \& Heinzel (2009). We processed the eclipse observations in RGB channels of the digital Canon camera and used the ratio between two channels (RGB signals are not calibrated), together with the $\mathrm{H} \alpha$ integrated intensity, to derive the electron density and the effective geometrical thickness along the line of sight.

\section{Summary of results}

We obtained 2D maps of the kinetic temperature and electron density of the studied prominence (Figure 2). The temperature varies between 6000 and $15000 \mathrm{~K}$ and is consistent with recent measurements of Park et al. (2013). The central part of the prominence has lower temperature than the edges, between 7000 and $8000 \mathrm{~K}$. The electron density 


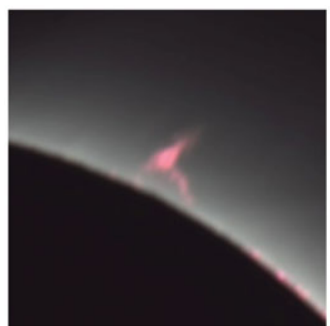

(a)

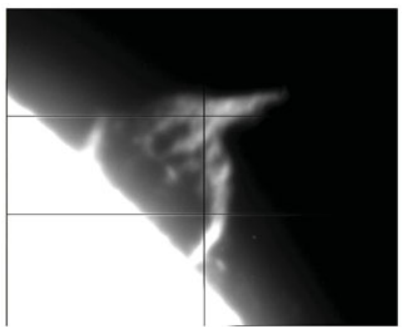

(b)

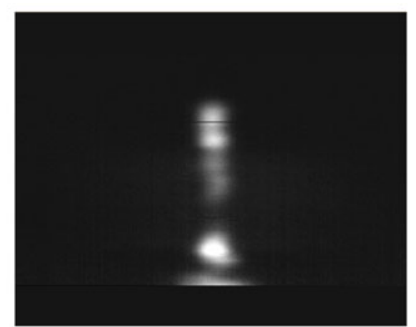

(c)

Figure 1. a)Total solar eclipse observed on August 1, 2008 at 11:05:29.6 UT in Mongolia with the studied prominence. b) The slit-jaw image of the studied prominence obtained at Ondřejov observatory on August 1, 2008 at 08.10.42.0 UT. c)The $\mathrm{H} \alpha$ line spectra along the vertical slit shown in (b).

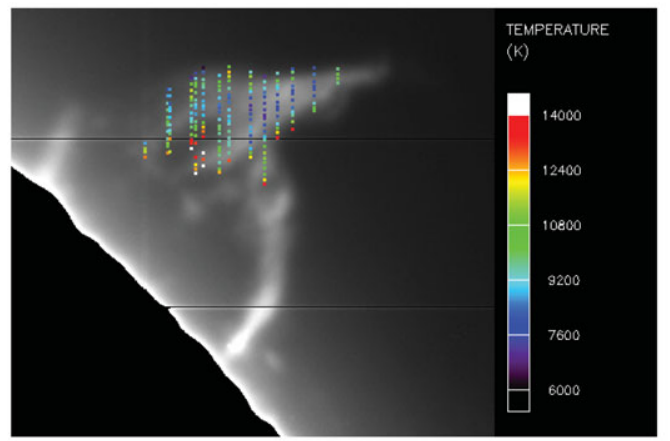

(a)

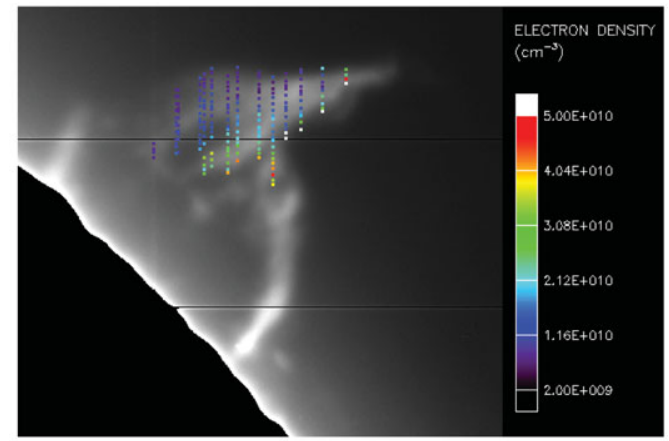

(b)

Figure 2. a) Map of the kinetic temperature of the studied prominence. b) Map of the electron density.

varies between $5 \times 10^{9}-10^{11} \mathrm{~cm}^{-3}$ and is quite comparable with the values from Bommier et al. (1994). The electron density is systematically increasing towards the bottom of the prominence which could be explained by an enhanced photoionization by the incident solar radiation. We also obtained 2D map of the effective thickness which varies between about 200 and $15000 \mathrm{~km}$. Typical value is around $10000 \mathrm{~km}$ and is decreasing towards the bottom. Using the obtained maps of the electron density and kinetic temperature we also computed the prominence gas pressures, assuming a uniform ionization degree of hydrogen $i=0.5$ in cool parts. The prominence seems to be a low-pressure structure with a typical gas pressure around $0.05 \mathrm{dyn} \mathrm{cm}^{-2}$.

New prominence observations obtained during solar eclipses or using the space coronagraphs are required together with simultaneous detection of the Balmer lines and other lines falling into the wavelength range of detected visible-line continuum.

\section{References}

Bommier, V., Landi Degl'Innocenti, E., Leroy, J.-L., \& Sahal-Brechot, S. 1994 Solar Phys., 154, 231

Heinzel, P., Gouttebroze, P., \& Vial, J. C. 1994, Astron. Astrophys. , 292, 656

Jejčič, S. \& Heinzel, P. 2009 Solar Phys., 254, 89

Park, H., Chae, J., Song, D., Maurya, R. A., Yang, H., Park, Y.-D., Jang, B.-H., Nah, J., Cho, K.-S., Kim, Y.-H., Ahn, K., Cao, W., \& Goode, P. R. 2013 Solar Phys., doi:10.1007/s11207013-0271-2 